\title{
Meditation Increases the Entropy of Brain Oscillatory Activity
}

\author{
Rocío Martínez Vivot, ${ }^{\mathrm{a}, \mathrm{b}, \mathrm{c} \dagger}$ Carla Pallavicini, ${ }^{\mathrm{a}, \mathrm{b}, \mathrm{d} \dagger}$ Federico Zamberlan, ${ }^{\mathrm{a}, \mathrm{b}}$ Daniel Vigo ${ }^{\mathrm{a}, \mathrm{c}}$ and Enzo Tagliazucchi ${ }^{\mathrm{a}, \mathrm{b} *}$ \\ ${ }^{a}$ National Scientific and Technical Research Council (CONICET), Buenos Aires, Argentina \\ ${ }^{\mathrm{b}}$ Departamento de Física, FCEyN, UBA, e Instituto de Física de Buenos Aires (IFIBA), Buenos Aires, Argentina \\ ${ }^{c}$ Instituto de Investigaciones Biomédicas UCA-CONICET (BIOMED), Buenos Aires, Argentina \\ ${ }^{\mathrm{d}}$ Fundación para la Lucha contra las Enfermedades Neurológicas de la Infancia (FLENI), Buenos Aires, Argentina
}

\begin{abstract}
We address the hypothesis that the entropy of neural dynamics indexes the intensity and quality of conscious content. Previous work established that serotonergic psychedelics can have a dysregulating effect on brain activity, leading to subjective effects that present a considerable overlap with the phenomenology of certain meditative states. Here we propose that the prolonged practice of meditation results in endogenous increased entropy of brain oscillatory activity. We estimated the entropy of band-specific oscillations during the meditative state of traditions classified as 'focused attention' (Himalayan Yoga), 'open monitoring' (Vipassana), and 'open awareness' (Isha Shoonya Yoga). Among all traditions, Vipassana resulted in the highest entropy increases, predominantly in the alpha and low/high gamma bands. In agreement with previous studies, all meditation traditions increased the global coherence in the gamma band, but also stabilized gamma-range dynamics by lowering the metastability. Finally, machine learning classifiers could successfully generalize between certain pairs of meditation traditions based on the scalp distribution of gamma band entropies. Our results extend previous findings on the spectral changes observed during meditation, showing how long-term practice can lead to the capacity for achieving brain states of high entropy. This constitutes an example of an endogenous, self-induced high entropy state. $\odot 2020$ IBRO. Published by Elsevier Ltd. All rights reserved.
\end{abstract}

Key words: meditation, EEG, entropy, alpha oscillations, gamma oscillations.

\section{INTRODUCTION}

The spectrum of human consciousness is rich and varied, yet the neural underpinnings of such diversity remain elusive (Koch et al., 2016). Recently, Carhart-Harris and colleagues introduced the hypothesis that the degree of entropy of spontaneous brain activity indexes the 'richness' of conscious subjective experience (Carhart-Harris et al., 2014). Entropy is a dimensionless quantity derived from the theory of statistical mechanics that can be interpreted as the degree of disorder present in a physical system (Ben-Naim, 2012). As expressed by the proponents of the hypothesis: "The great merit of applying the measure of entropy in cognitive neuroscience is that it is uniquely adept at bridging the physical and subjective divide; mere flip sides of the same coin - but different sides nonetheless". According to this proposal, low-

\footnotetext{
*Correspondence to: E. Tagliazucchi, National Scientific and Technical Research Council (CONICET), Buenos Aires, Argentina.

E-mail address: tagliazucchi.enzo@googlemail.com (E. Tagliazucchi).

$\dagger$ These authors contributed equally to this work Abbreviations: EEG, electroencephalography; FA, focused attention; HT, Himalaya Yoga; OM, open monitoring; PET, positron emission tomography; RF, random forests; SE, sample entropy; VIP, Vipassana.
}

entropy brain states present a reduced repertoire of potential configurations, and thus are subjectively experienced as states of diminished conscious awareness (Schartner et al., 2015; 2017b; Hudetz et al., 2016; Liu et al., 2018). Conversely, high-entropy brain states are experienced as content-rich, but at the same time as unpredictable and therefore uncertain (Tagliazucchi et al., 2014; Cavanna et al., 2017; Carhart-Harris, 2018), and thus enabling subjective experiences beyond the scope of everyday awareness. As an example, the acute effects of serotonergic psychedelics (i.e. serotonin 5- $\mathrm{HT}_{2 \mathrm{~A}}$ receptor agonists), glutamatergic dissociatives (i.e. NMDA receptor antagonists) (Schartner et al., 2017a; Viol et al., 2017; 2019) and a nonselective adenosine antagonist stimulant (caffeine) (Chang et al., 2018) have been linked to high-entropy brain oscillations or, equivalently, to reduced levels of compressibility.

In the information-theoretical sense, entropy represents the amount of information needed to specify the microstate of the system or, equivalently, its level of 
uncertainty and unpredictability. ${ }^{2}$ The biological meaning of brain entropy depends upon the experimental variable under study. The large-scale oscillations in scalp potential that are measured using electroencephalography (EEG) are frequently employed as markers of physiological, pathological and pharmacologically-induced global brain states (e.g. sleep, epilepsy, coma, anesthesia). Thus, the entropy of such global brain oscillations could approximate the uncertainty of the distribution of potential brain configurations, yielding an objective marker of the subjectively felt 'richness' of conscious experience.

We propose that the brain state achieved by expert meditators is associated with increased entropy of brain oscillatory activity. This hypothesis is supported by the observation that the extended practice of certain meditation traditions is associated with an enlarged repertoire of subjective experiences in comparison to ordinary conscious wakefulness (Brandmeyer et al., 2019). In particular, many of these features are common to the acute effects of serotonergic psychedelics. These features include a shift towards positive mood and emotions, increased cognitive flexibility and emotional dysregulation or mood lability, altered self-awareness, a state of a 'dreamlike' quality, and the collapse of the intentional structure of subject/object (non-dual awareness) (Carhart-Harris and Nutt 2014; Travis, 2014; Nichols, 2016; Nour and Carhart-Harris, 2017; Millière et al., 2018). Both meditation and the use of serotonergic psychedelics are frequently associated with the intention of obtaining 'insights' about the nature of 'objective reality' and the self (Barrett and Griffiths, 2018). Furthermore, evidence suggests that certain meditative practices (Strauss et al., 2014; Haukaas et al., 2018) and serotonergic psychedelics (Griffiths et al., 2016; Carhart-Harris et al., 2017, 2018; Dos Santos et al., 2018; GarciaRomeu and Richards, 2018; Palhano-Fontes et al., 2018) can lead to prolonged positive effects in patients suffering from depression and anxiety, raising the possibility of increased brain entropy as a common mechanism underlying these effects (Carhart-Harris, 2018).

It is now widely recognized that, by itself, the term 'meditation' is non-specific, and that it has been frequently applied to heterogeneous practices, both in terms of cultural background and the instructions followed to meditate (Braboszcz et al., 2017). To evaluate our hypothesis while also accounting for this heterogeneity, we investigated EEG data acquired from three groups of expert meditators following different traditions: Himalaya Yoga (HT), Vipassana (VIP) and Isha Yoga (SNY). These traditions can be placed along a continuum ranging from 'focused attention' (FA) to 'open monitoring' (OM) meditation (Lutz et al., 2008; Lippelt et al., 2014). HT uses a mantra to maintain attentional focus and can be classified as FA. SNY meditators practiced Shoonya meditation, consisting of a process of conscious 'non-doing' while remaining unresponsive to internal or external stimuli, which can be classified as OM. In between these two

${ }^{2}$ More precisely, information is proportional to Gibbs entropy, $G \propto \sum_{i} p_{i} \log \left(p_{i}\right)$, where $p_{i}$ represents the probability of the system residing in its $\mathrm{i}$-th microstate. The original definition (given by Boltzmann) is recovered when all $p_{i}$ are equal. extremes, VIP requires practitioners to sustain their focus ('samatha') in the process of mentally scanning bodily sensations; however, a key component of this meditation is the insight (or meta-awareness, 'vipasyana') of distracting elements, which must be identified and then released to continue with the process of FA (Lutz et al., 2007).

The local entropy of band-specific oscillatory activity at each scalp electrode was complemented with the analysis of two global metrics: the long-range coherence of oscillations, and its fluctuations over time (i.e. metastability). Both metrics are motivated by theoretical accounts of consciousness and have been empirically shown to depend on the level of awareness (CarhartHarris et al., 2014; Schartner et al., 2015, 2017a,b).

\section{EXPERIMENTAL PROCEDURES}

We investigated a publicly available EEG dataset. Further information on the experimental procedures can be found in a previous publication (Braboszcz et al., 2017). All codes used in this manuscript can be found in https:// github.com/enzotagliazucchi/meditation-entropy.

\section{Participants}

Thirty controls (CTR), $27 \mathrm{HT}$ meditators, $20 \mathrm{VIP}$ meditators and 27 SNY meditators were recruited based on age, gender and years of practice. The control subjects were chosen for inclusion in this study based on age, gender, and the absence of meditation practice. The experiment was approved by the local ethics committee and by the ethics committee of the University California San Diego. The experiments were carried out at the Meditation Research Institute (MRI) in Rishikesh, India. Groups were matched by age and gender, resulting in 16 controls ( $45 \pm 10$ years, five females), $16 \mathrm{HT}$ meditators (43 \pm 12 years, 2 females), 16 SNY meditators ( $40 \pm 10$ years, 2 females) and 16 VIP meditators ( $47 \pm 15$ years, five females). A single group of subjects acted as control for all three meditation traditions.

\section{Experimental design}

All participants were instructed to keep their eyes open and choose between sitting on a blanket on the floor or on a chair. The experiment was divided into two sessions lasting $20 \mathrm{~min}$ each, one labeled as 'Meditation' and the other as 'Instructed Mind Wandering'. The order of the sessions was counterbalanced between participants to prevent order effects. In the 'Meditation' condition, participants prepared for their meditation practice (focusing on breath or on exhalation/inhalation) during $10 \mathrm{~min}$ and were subsequently instructed to engage in meditation during the last $10 \mathrm{~min}$. Controls performed the same instruction given to the meditators throughout the $20 \mathrm{~min}$ of the condition. In the 'Instructed Mind Wandering' condition, participants were instructed to remember autobiographical events from childhood up until the recent past. The present analysis is based on comparing the second half of the 'Meditation' condition between meditators and controls. As additional checks, 
we also compared the 'Instructed Mind Wandering' condition of the controls vs. the 'Meditation' condition of the meditators, and the baseline condition (preparation for meditation) between both groups.

\section{EEG data acquisition}

EEG data was recorded using a $64+8$ channels Biosemi Active-Two amplifier system with a 10-20 Headcap standard 64-channel cap. Right and left mastoid electrodes as well as vertical and horizontal electrooculogram (EOG) were recorded. The experimental room was soundproof and the floor was electrically shielded and grounded. All electrodes were kept within $50 \mathrm{mV}$ offset of the Biosemi system metric for measuring impedance.

\section{EEG data processing}

Using EEGLAB (version 12) data were first referenced to the right mastoid and down-sampled from $1024 \mathrm{~Hz}$ to $256 \mathrm{~Hz}$. A high-pass filter with $1 \mathrm{~Hz}$ cutoff (infinite response filter), a transition bandwidth of $0.3 \mathrm{~Hz}$, and an order of six was applied to the EEG time series. EEGLAB automated artifact rejections routines were also applied. A manual procedure described with detail in a previous publication (Braboszcz et al., 2017) was followed to reject $8 \mathrm{~s}$. epochs with very high/low frequency content. Bad electrodes (0-18 bad electrodes per subject, average of five electrodes per subject) were identified visually and removed from the analysis. The Infomax independent component analysis algorithm was applied to reject artifacts related to eye movement and muscle activity.

\section{Bandpass filtering and the Hilbert transform}

We focused our analyses on the following canonical frequency bands: delta $(1-4 \mathrm{~Hz})$, theta $(4-8 \mathrm{~Hz})$, alpha $(8-12 \mathrm{~Hz})$, beta $(12-20 \mathrm{~Hz})$, low gamma $(20-60 \mathrm{~Hz})$ and high gamma $(60-100 \mathrm{~Hz})$. Our choice of computing the entropy of the envelope of band-specific oscillations is based on the following two considerations. First, narrow frequency bands have been linked to specific brain functions, cognitive domains and, in particular, to the meditative state (Buzsaki, 2006; Braboszcz et al., 2017). Second, it is unclear how the spectral content of broadband signal fluctuations biases entropy estimates or, equivalently, to which extent the entropy of broadband signals provides information complementary to spectral analysis.

For each EEG channel, we applied a 4th order Butterworth bandpass filter to obtain the narrow band signals in the above-mentioned ranges. We then obtained the analytical representation of the filtered time series using the Hilbert transform. As illustrated in Fig. 1A, given a real-valued signal $x(t)$, the Hilbert transform yields a complex representation (analytic signal) given by $z(t)=u(t)+i v(t)=x(t)+i H(x(t))$, with $H(x(t))$ indicating the Hilbert transform of the real-valued signal $x(t)$. From this representation, the instantaneous amplitude can be computed as $\sqrt{u(t)^{2}+v(t)^{2}}$, and the instantaneous phase as $\tan ^{-1}\left(\frac{v(t)}{u(t)}\right)$.

\section{Sample entropy (SE)}

The SE is an estimate of the complexity (or, equivalently, of the regularity or predictability) of a time series that is based on counting repetitions of patterns in the data (Richman and Moorman, 2000). SE measures the probability of observing similar (within a certain tolerance bound $[r]$ ) patterns of a fixed length in the time series, compared to the result obtained from the same computation based on patterns of length increased by one sample. The length of the patterns is determined by the embedding dimension $(m)$. In contrast to recent publications, we chose SE instead of the Lempel-Ziv (LZ) complexity, since LZ estimates information in the algorithmic sense (i.e. compressibility) and requires arbitrary binarization of the data (Schartner et al., 2015), while SE can be applied to continuous signals and yields entropy estimates more directly related to the statistical mechanical (Gibbs') and information theoretical (Shannon's) definitions of entropy.

The detailed procedure followed to compute SE is explained in the Appendix. The key computation is $\mathrm{SE}=\log \left(\frac{\# S_{m+1}(r)}{\# S_{m}(r)}\right)$, where $\# S_{m}$ and $\# S_{m+1}$ represent the number of template vectors of embedding dimension $m$ and $m+1$, respectively, within tolerance $r$. Following previous publications (e.g. Al-Angari and Sahakian, 2007; Bruce et al., 2009; Song et al., 2012; Zhang and Zhou, 2012; Yentes et al., 2013), we set the value of the embedding dimension to $m=2$. It is also customary to express the tolerance $r$ as proportional to the standard deviation (SD) of the time series; here we set $r=0.2 \times \mathrm{SD}$ (see the Appendix for further details). Finally, to reduce the impact of autocorrelations in the data, we considered $\tau=32 \mathrm{~ms}$.

\section{Metastability and coherence}

Consider $N$ coupled oscillators (in this case, each of the 64 bandpass-filtered EEG channels can be conceptualized as an oscillator), each with its own characteristic oscillation frequency $\omega_{i}$. The instantaneous amount of synchrony between the ensemble of oscillators can be measured with the Kuramoto order parameter. The order parameter $R(t)$ ranges between 0 and 1 , indicating minimum and maximum synchrony, respectively (Shanahan, 2010). The metastability $(\mathrm{K})$ is computed as the temporal variance of $R(t)$, and indicates the amplitude of the dynamical repertoire of the oscillators (i.e. the occurrence of transient synchronization and de-synchronization over time). The coherence $\mathrm{C}$ is computed as the mean of $R(t)$, and indicates the average level of synchronization between all oscillators. The procedure followed to compute $\mathrm{K}$ and $\mathrm{C}$ is outlined in Fig. $1 \mathrm{C}$, and explained with further detail in the Appendix.

\section{Random forest (RF) classifiers}

To evaluate whether changes in SE represent signatures specific to each of the meditative traditions, we used this data to train and evaluate a class of machine learning classifiers known as RF (Breiman, 2001). RF are built on the simpler decision tree classifier, in which thresholds 


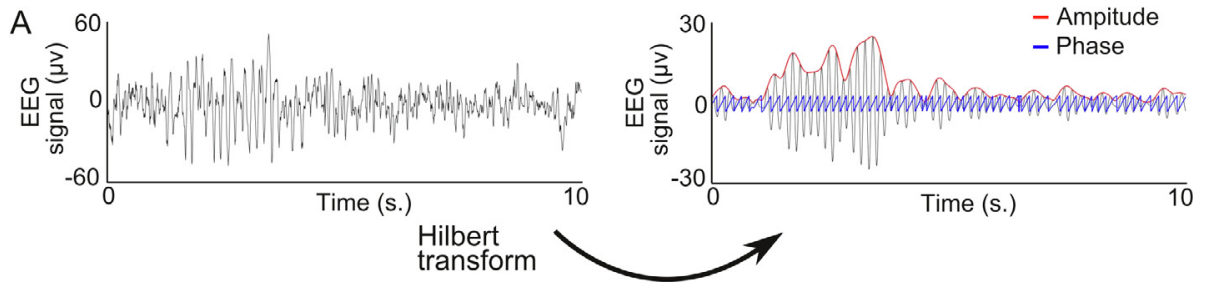

B
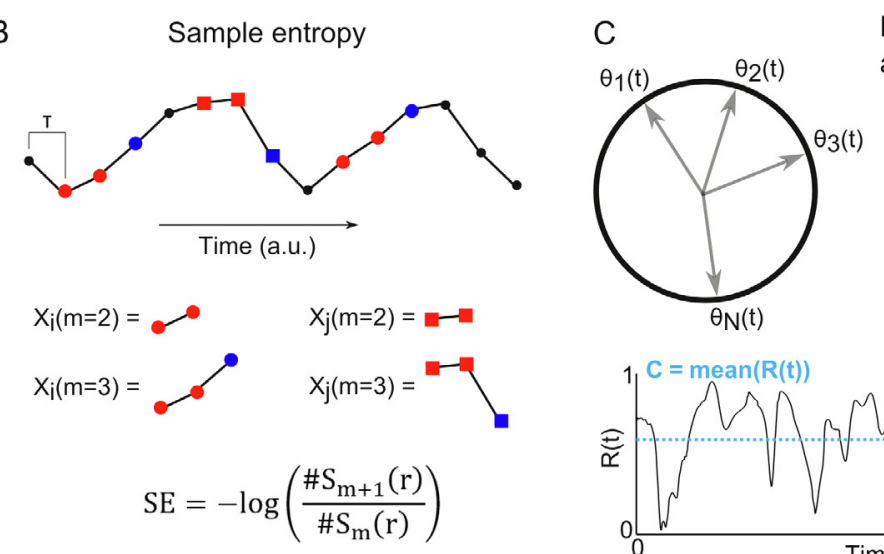

Metastability and coherence
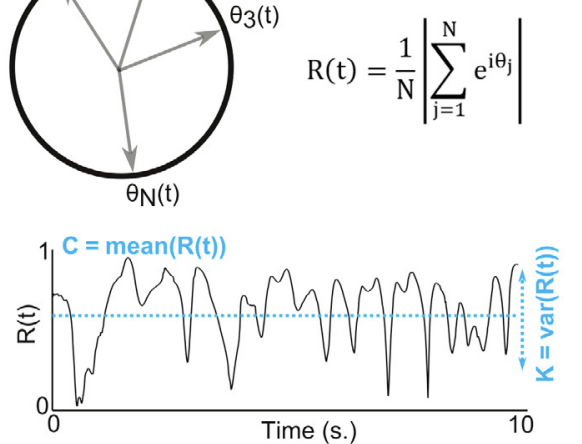

D

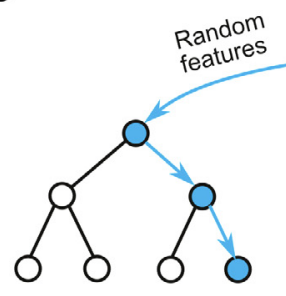

Class A

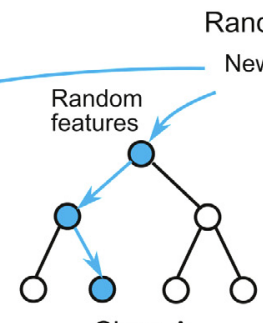

Class A

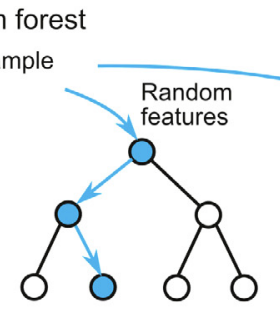

Class A

Majority voting: Class $\mathrm{A}$

Fig. 1. Overview of the methods. (A) Bandpass filtering and the application of the Hilbert transform to obtain the instantaneous amplitude and phase of the EEG signal. (B) Example of the SE algorithm with embedding dimension $m=2$. Two template vectors of length $m$ are shown, $X_{2}(i=2)$ and $X_{2}(i=6)$, together with the associated template vectors of embedding dimension $m+1$. The sample entropy is obtained by counting the number of pairs of template vectors (for $m$ and $m+1$ ) whose distance is below the tolerance r, i.e. $\# S_{m}(r)$ and $\# S_{m+1}(r)$, and then applying Eq. (1). (C) Definition of the instantaneous synchronization $R(t)$ of a set of $N$ oscillators with phases $\theta_{i}(t)$. A representative time course of $R(t)$ is shown, providing an example of the definition of the coherence $\mathrm{C}$ and the metastability $\mathrm{K}$ as the mean and variance of $R(t)$, respectively. (D) Diagram of the RF algorithm. For each training sample, a random subset of features is selected and then a decision tree is trained using these features. The class of a new sample is predicted by evaluating the decision trees trained with the chosen subset of features, and subsequently applying a voting or majority rule (in this example the voting predicts class $\mathrm{A}$ ).

are introduced for each feature so that the training set is split in two at every node of the tree. The thresholds are selected to maximize a measure of sample purity resulting from the division; the highest possible purity value is obtained when all samples belonging to one class are assigned to one side of the division, and vice versa. If this is not the case, the next feature is evaluated and the threshold is recursively determined until the highest purity value is obtained or all features are exhausted.

The RF algorithm is based on training a relatively large number of decision trees (in this case 1.000 trees) so that the impact of noise at a particular feature is attenuated. Furthermore, to reduce the influence of noisy or unreliable features, each individual decision

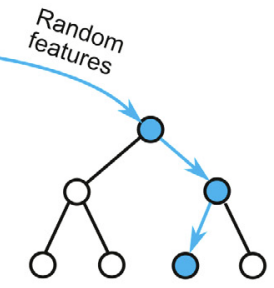

Class B tree is based on a random selection of features from the original data. Here we follow the rule of thumb of considering $\sqrt{p}$ features, where $p$ is the original number of features. For less than 100 features, this choice approximates very well the original value of $\left\lfloor 1+\log _{2}(p)\right\rfloor$ proposed by L. Breiman in his original publication of the algorithm (Breiman, 2001). Once trained, to predict the class for a new sample a probability is estimated by counting how many trees predict each class, divided by the total number of trees. The final class is decided by a majority vote. This procedure is illustrated in Fig. 1D.

For each frequency band we considered 64 features corresponding to the SE entropy values at each EEG electrode location, and trained 200 independent RF classifiers to distinguish CTR from HT, VIP and SNY (Abraham et al., 2014). As a measure of classifier accuracy, we obtained the area under the ROC curve (AUC). For each classifier, we applied a stratified split into two equally sized sets (training and testing). We also applied the same procedure after randomly shuffling the labels of the samples to evaluate the statistical significance of the AUC. An empirical p-value was constructed by counting how many times the AUC obtained from data with shuffled labels exceeded the AUC obtained from the unshuffled data.

Finally, we investigated the generalization accuracy by training the RF classifiers using data from one binary decision problem (e.g. CTR vs. VIP) and then evaluating the AUC obtained from applying them to a different binary decision problem (e.g. CTR vs. HT).

\section{Statistical analysis}

We applied the Kruskal-Wallis (KW) test, a nonparametric version of analysis of variance (ANOVA), to assess the effects of the experimental group (CTR, HT, VIP and SNY) on SE (averaged across all channels), C, and $K$. Whenever a significant effect of group was detected, we conducted post-hoc non-parametric twosample Wilcoxon signed-rank tests between data from CTR and from each meditative tradition. Effect sizes of the Wilcoxon tests were computed as $r=\frac{z}{\sqrt{n_{1+n_{2}}}}$, where 
A

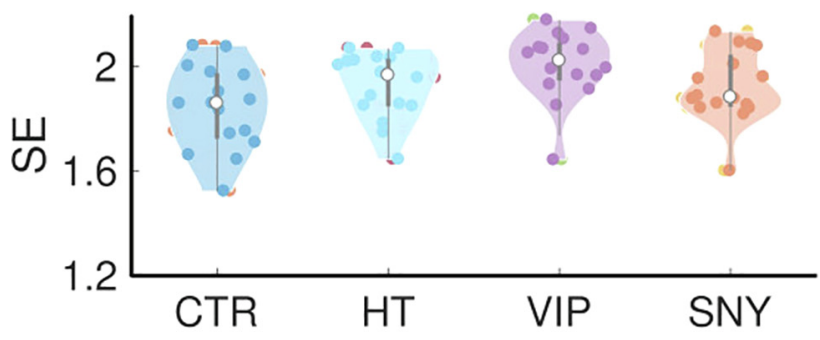

B

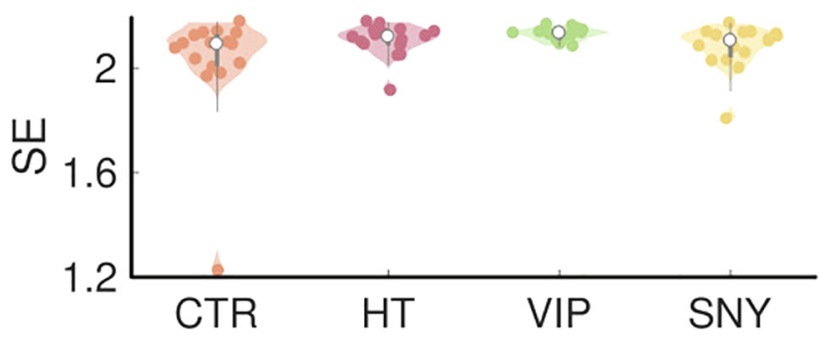

C

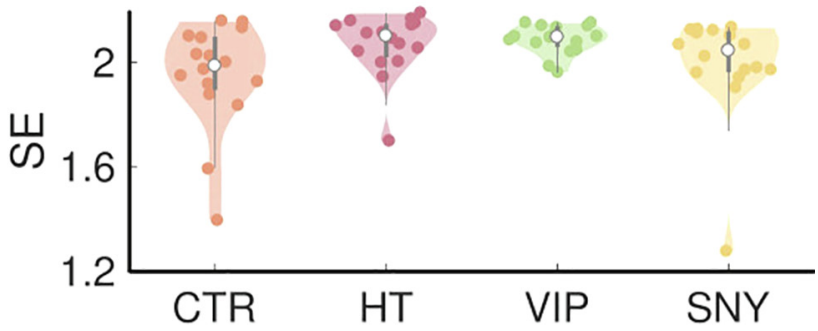

Fig. 2. Effect of meditation on the entropy of brain oscillations. The meditative state changes the entropy of brain oscillations in the alpha (panel A), low gamma (panel B) and high gamma (panel C) bands. The violin plots display the distribution of SE values (averaged for all electrodes) across individual participants. Statistics are reported in the Results section. $n_{1}=n_{2}=16$ (the sizes of the samples being compared) (Rosenthal et al., 1994). Effect sizes of Kruskal-Wallis tests were measured in terms of $\eta^{2}$ (Lakens, 2013). Multiple comparisons (e.g. scalp distributions in Fig. 3, or RF classifier generalizability in Fig. 4) were controlled using Benjamini-Hochberg's method for false discovery rate $(F D R)$ allowing a rate of false positives of 0.05 . Only the frequency bands surviving multiple comparisons correction (FDR-corrected Kruskal-Wallis tests) are shown in Figs. 2 and 5 , with the exception of the metastability $(\mathrm{K})$ in the low gamma band, which showed a trend towards significance.

\section{RESULTS}

\section{SE of narrow band signal envelopes}

We first computed the SE of narrow band EEG signal envelopes for all subjects, electrodes and frequency bands. For the first analysis we used the second $10 \mathrm{~min}$ of the 'Meditation' condition, which corresponded to the instruction of focusing on breath (exhalation/inhalation) for the controls, and to engage in their practice for the meditators. We $c$ of group on the average SE $(H[3]$ $=8.86, p \leq 0.05, \eta^{2}=0.42$, FDR-corrected) for the alpha $(8-12 \mathrm{~Hz})$, low gamma $(20-60 \mathrm{~Hz})(\mathrm{H}[3]=9.56$, $p \leq 0.05, \eta^{2}=0.44$, FDR-corrected) and high gamma $(60-100 \mathrm{~Hz}) \quad\left(\mathrm{H}[3]=8.5, \quad p \leq 0.05, \quad \eta^{2}=0.41, \quad\right.$ FDRcorrected) frequency bands. Post-hoc non-parametric Wilcoxon tests established that, at $p \leq 0.05$, FDRcorrected, only the comparison Vipassana $>$ controls (VIP $>$ CTR) was significant $(Z=2.65, r=0.46$, $p \leq 0.005$ ), except for the high gamma band, for which we observed that Himalaya Yoga $>$ controls $(H T>C T R) \quad(Z=2.12, \quad r=0.38, \quad p \leq 0.05) \quad$ and VIP > CTR $\quad(Z=2.28, \quad r=0.40, \quad p \leq 0.05)$ were significant. The average SE values are shown in Fig. 2.

Next, we compared the 'Instructed Mind Wandering' condition for the controls vs. the 'Meditation' condition for the meditators. We observed a significant effect of group on the average SE $(H[3]=11.71, p \leq 0.05$, $\eta^{2}=0.49$, FDR-corrected) for the alpha $(8-12 \mathrm{~Hz})$, the low gamma $(20-60 \mathrm{~Hz}) \quad(\mathrm{H}[3]=10.5, \quad p \leq 0.05$, $\eta^{2}=0.47, \quad$ FDR-corrected $)$ and high gamma $(60-$ $100 \mathrm{~Hz}) \quad\left(\mathrm{H}[3]=9.94, \quad p \leq 0.05, \quad \eta^{2}=0.45, \quad\right.$ FDRcorrected) frequency bands. Post-hoc non-parametric Wilcoxon tests established that, at $p \leq 0.05$ FDRcorrected, the comparison VIP $>$ CTR was significant for the alpha $(Z=2.92, \quad r=0.51, \quad p \leq 0.005)$, low gamma $(Z=2.77, r=0.48, p \leq 0.01)$ and high gamma ( $Z=2.65, r=0.46, p \leq 0.01)$ frequency bands.

We did not observe a
A

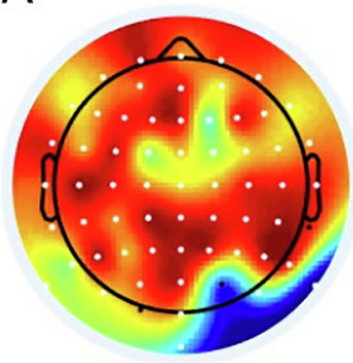

B

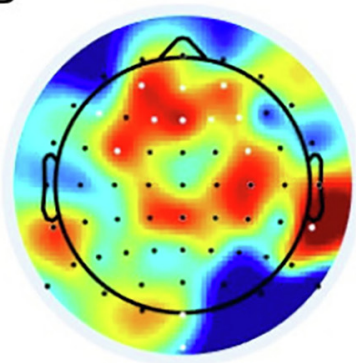

C

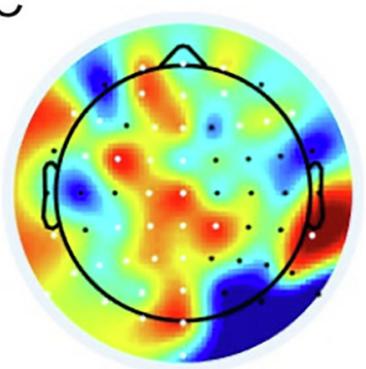

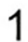

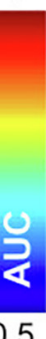

Fig. 3. Localization of entropy changes. Topographic distribution of higher SE values in VIP relative to CTR for the alpha (panel A), low gamma (panel B) and high gamma (panel C) bands. Electrodes in white represent significantly higher SE ( $p \leq 0.05$, Wilcoxon test, FDR-corrected). The color scale indicates the effect size in terms of the area under the receiving operator characteristic curve (AUC). significant effect of group on SE values for any frequency band when comparing the SE values obtained at the baseline condition (i.e. preparation for meditation).

The topographic distribution of electrodes where SE increased in VIP vs. CTR is shown in Fig. 3. Significant electrodes (Wilcoxon test, FDR-corrected, $p \leq 0.05$ ) are represented in white. The color scale indicates the effect size in terms of the area under the receiving operator characteristic curve (AUC). We found widespread SE increases in the 

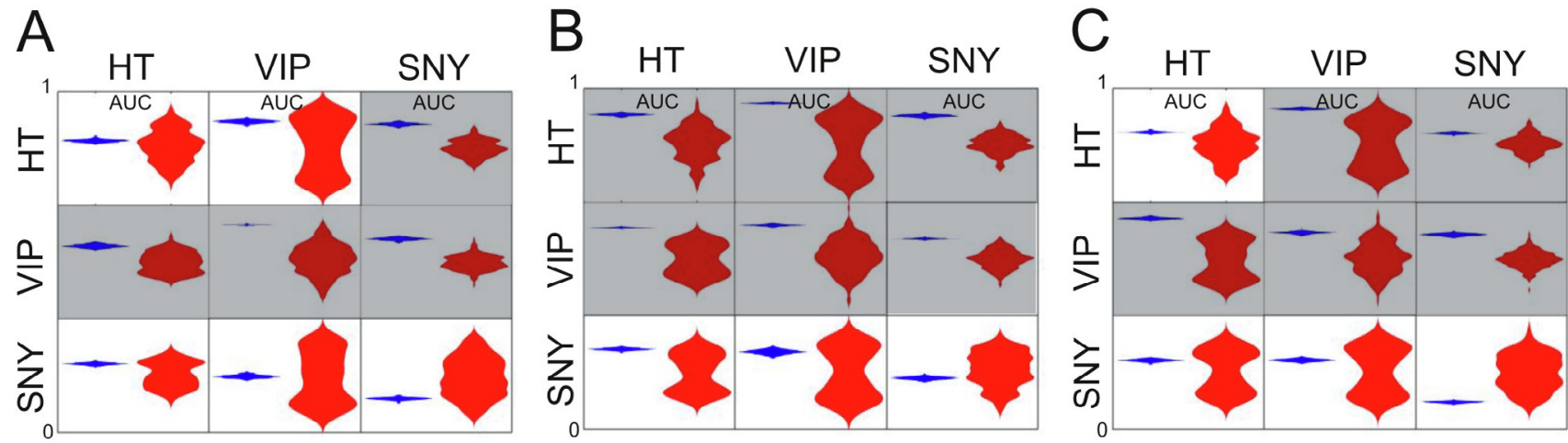

Real labels

\section{Shuffled labels $\square p<0.05$}
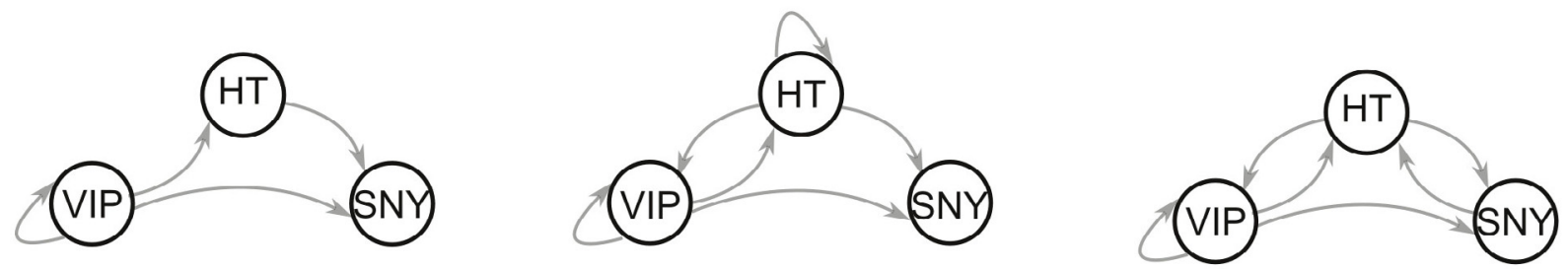

Fig. 4. Generalization of entropy changes between meditation traditions. Multivariate analysis based on training and testing RF classifiers using the SE values from the 64 EEG channels in the alpha (panel A), low gamma (panel B) and high gamma (panel C) bands. Upper panel: RF classifiers were trained to distinguish the meditation tradition (row) from the CTR group. Afterwards, they were evaluated in the problem of distinguishing the meditation tradition in the columns from CTR. Entries in the diagonal correspond to training and evaluating the classifiers using a train/test split of the same dataset. The entries show the distribution (violin plots) of AUC values obtained from unshuffled (blue) shuffled (red) data. Opaque entries indicate that the empirical $p$-value (obtained from comparing both AUC distributions) is $\leq 0.05$ (FDR-corrected). Bottom panel: each node represents a meditation tradition and arrows indicate that the RF classifier trained to distinguish the tradition at the source node from CTR successfully distinguished the tradition at the target node from CTR. E.g. the arrows from VIP to the other nodes indicate that RF classifiers trained to distinguish VIP from CTR successfully generalized to the other two meditation traditions. (For interpretation of the references to colour in this figure legend, the reader is referred to the web version of this article.)

alpha band, predominantly frontal SE increases in the low gamma band, and central and frontal SE increases in the high gamma band.

\section{Multivariate classifiers applied to SE topographies}

We then investigated whether a multivariate analysis based on RF classifiers could distinguish the meditative state from the control condition for all groups of
A
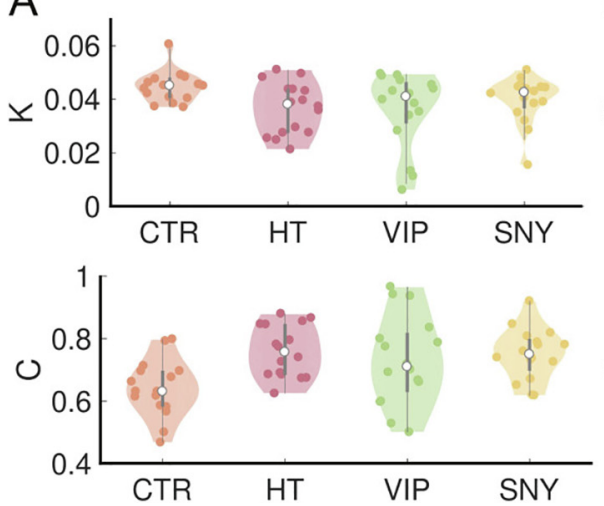

B
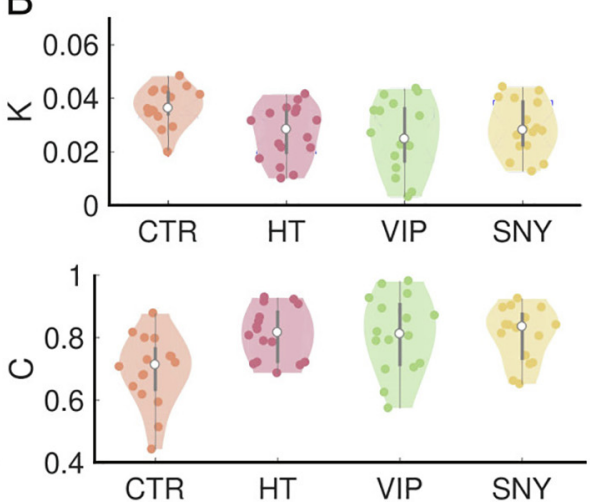

Fig. 5. Effect of meditation on the dynamics of the Kuramoto order parameter. The meditative state changes the coherence $(C)$ and metastability $(K)$ of low gamma (panel A) and high gamma (panel B) band oscillations. The violin plots display $\mathrm{K}$ and $\mathrm{C}$ distributions across individual participants. Statistics are reported in the Results section. meditators. RF classifiers were based on the combined SE values from all 64 EEG channels at the frequency bands for which we observed a significant effect of group (alpha, low and high gamma). We also evaluated whether a classifier trained to distinguish a certain meditative state (e.g. HT) from CTR could successfully generalize to the problem of distinguishing the meditative state of another group (e.g. VIP) from the CTR. This analysis is relevant to identify potential 'core' features that are common to different meditation traditions.

The results of this analysis are shown in Fig. 4. In the upper panel the rows correspond to the group used for training the RF classifiers, and columns correspond to the group used to evaluate their performance. Diagonal entries correspond to training and evaluating RF classifiers using the same group (i.e. half of the data used for training and half for evaluation). Within each entry, the blue/red violin plots represent the results obtained using unshuffled/shuffled class labels. Shaded entries indicate that the corresponding 
AUC was greater than chance $(p<0.05$, FDRcorrected). The lower panel in Fig. 4 shows all training/ evaluation pairs yielding AUC significantly greater than chance.

In all cases, it was possible to distinguish with high accuracy the VIP from CTR groups. This was not the case for other meditative traditions, except for HT in the low gamma band; however, we note that the RF classifiers trained to distinguish VIP from CTR successfully generalized to the other two meditation traditions for all three frequency bands. These results are consistent with those presented in Figs. 2 and 3, and suggest that, even though increased SE is characteristic of VIP, the topographic distribution of SE can be informative of features that are common to other meditative traditions.

\section{Coherence and metastability}

Finally, we investigated the coherence $C$ across all EEG electrodes, computed as the temporal average of the synchrony $R(t)$. We also obtained the metastability $\mathrm{K}$ from the variance of $R(t)$. The upper/lower panels of Fig. 5 show K/C, respectively, for all the experimental groups. We assessed the effect of the group on $\mathrm{K}$ in the low $\left(H[3]=5.97, p \leq 0.1, \eta^{2}=0.33\right)$ and high gamma bands $\left(H[3]=9.95, p \leq 0.05, \eta^{2}=0.45\right)$, as well as the effect of the group on $C$ in the low $(H[3]=12.03$, $\left.p \leq 0.01, \eta^{2}=0.50\right)$ and high gamma bands $(H[3]$ $=11.2, p \leq 0.01, \eta^{2}=0.48$ ).

Post-hoc non-parametric Wilcoxon tests revealed that in the low gamma band $\mathrm{K}$ decreased during meditation for the comparisons HT $<$ CTR $(Z=2.24, p \leq 0.01$, $r=0.39$ ) and Isha Yoga $>$ controls (SNY $<$ CTR) $(Z=1.16, p \leq 0.05, r=0.29)$; in the high gamma band for comparisons HT $<$ CTR $(Z=2.27, \quad p \leq 0.005$, $r=0.39), \quad \mathrm{VIP}<\mathrm{CTR}(Z=2.35, p \leq 0.01, r=0.41)$ and $\mathrm{SNY}<\mathrm{CTR} \quad(Z=2.31, \quad p \leq 0.01, \quad r=0.40)$. Conversely, in the low gamma band $C$ increased for HT $>$ CTR $(Z=3.10, p \leq 0.001, r=0.54)$, VIP $>$ CTR $(Z=1.90, p \leq 0.05, r=0.33)$, SNT $>$ CTR $(Z=3.03$, $p \leq 0.001, r=0.53$ ); and in the high gamma band $C$ increased for HT $>$ CTR $(Z=2.80, p \leq 0.005$, $r=0.49), \operatorname{VIP}>$ CTR $(Z=2.35, p \leq 0.01, r=0.41)$, SNY $>$ CTR $(Z=2.88, p \leq 0.005, r=0.51)$.

\section{DISCUSSION}

Previous studies reported changes in local oscillatory power and long-range coherence in different meditative practices that were neuroanatomically consistent with the reported subjective experience, e.g. increased occipital gamma has been consistently linked to changes in visual imagery (Lutz et al., 2004, 2007; Braboszcz et al., 2017; van Lutterveld et al., 2017; Kakumanu et al., 2018; Luft et al., 2019). The heterogeneity found in these reports highlights the ambiguous nature of the term 'meditation' (Brandmeyer et al., 2019; Schoenberg and Vago, 2019). Our analyses extended previous findings by showing how meditation can increase signal complexity in the alpha and gamma bands, as indexed by SE. In particular, SE changes in
VIP appeared as stereotypical, in the sense that they allowed machine learning classifiers to correctly generalize to the problem of detecting other meditative states from EEG signal entropy. Increased alpha and gamma power envelope entropy was observed for Vipassana meditation vs. controls, both when comparing meditation vs. focus on breathing and instructed mind wandering in the group of matched controls. However, we did not observe differences when comparing the 'focus on breathing' condition between meditators and controls, suggesting that our results are an active manifestation of engagement in meditation and do not reflect a trait effect.

The analyses conducted in the present work have relatively few antecedents. A previous study reported decreased permutation entropy (a method closely related to SE) during the practice of insight meditation (Vipassana) (Venugopala et al., 2017), which was also associated with increased levels of synchronization between the signals registered at the EEG electrodes. Increased gamma synchronization during the meditative state is consistent with our findings (Lutz et al., 2004). However, we found the opposite result for entropy (i.e. increased) when compared to the result reported by Venugopala and colleagues (i.e. decreased). We note that this discrepancy could be attributed to the fact that we did not investigate the entropy of the broadband EEG signal, but of the envelopes of narrow band oscillations. As mentioned earlier, in contrast to previous studies, our method allows to disentangle changes in the entropy of the envelope (possibly related to the size of cell assemblies whose activity oscillates at the carrier frequency) from the spectral content of the broadband signal, which contains unspecific contributions of cell assemblies oscillating at very different frequencies (i.e. $1 / \mathrm{f}$ spectrum).

The frequency bands where we found the most salient increases in entropy during meditation are consistent with the previous report by Braboszcz and colleagues comparing EEG spectral power between meditators and controls (Braboszcz et al., 2017) using the same dataset we analyzed. They reported increased occipital (Oz) EEG power for all meditation traditions vs. controls in the alpha $(7-11 \mathrm{~Hz})$ and gamma $(60-110 \mathrm{~Hz})$ ranges; see Fig. 3 in Braboszcz et al. (2017). Gamma band increases were located in occipito-parietal scalp positions, while alpha band increases were seen across all electrodes; see Figs. 4 and 6 in Braboszcz et al. (2017), respectively. These results are consistent with the topographic localization of increased alpha and gamma oscillation envelope entropies shown in Fig. 3.

Our results are also consistent with the observation of increased long-range coherence in the gamma (25$56 \mathrm{~Hz}$ ) band during the meditative state of Buddhist practitioners of the Nyingmapa and Kagyupa traditions. ${ }^{3}$ Vipassana meditation was reported to increase local

\footnotetext{
${ }^{3}$ These traditions differ from Vipassana in their instructions and objectives, and are described as 'loving-kindness' and 'compassion' meditation, with the objective of achieving a state of 'unconditional loving-kindness and compassion, resulting in unrestricted readiness and availability to help living beings'.
} 
gamma (35-45 Hz) power in parieto-occipital scalp regions (Cahn et al., 2010), overlapping with those reported in Fig. 3. Gamma band oscillations are implicated in several high-order cognitive processes such as attention, working memory, learning, and consciousness (Fries et al., 2007; Fries, 2009). They have also been postulated as a plausible neurobiological mechanism to solve the 'binding problem' (i.e. the integration of information between spatially distributed cortical regions) (Nikolić et al., 2013). In contrast with our results, Lutz et al. discuss a number of reports implicating slow oscillations with the meditative state (Lutz et al., 2007). However, and as also noted by the authors, these results could be biased by two fundamental factors: (1) fast oscillations were not investigated and (2) the studies were based on FA meditation, which could engage top-down control mechanisms related to the onset of slower brain oscillations.

Because the neurophysiological correlates of meditation have been historically confounded by several factors, the comparison of our results with the previous literature is difficult, especially so given that there are virtually no antecedents of articles investigating the entropy of band-specific oscillations. A major source of confusion is the nature of the meditative traditions that are investigated. Our unidimensional classification of meditation traditions from FA to OA is only one among other characterizations. As stated by Lutz et al. (2004), the EEG gamma band has not been measured in most electrophysiological studies of the meditative state. This is supported by a recent review and meta-analysis article by Thomas and Cohen (2014) (see Tables 2, 3, and 4 for FA, OA and transcendental meditation, respectively). It is apparent that FA studies generally reveal increases in the theta and alpha bands, while the results are less homogeneous for OM meditation.

Beyond the similarities in their phenomenology, both psychedelics and the meditative state have been investigated using $\mathrm{fMRI}$ to reveal decreased activity in the posterior cingulate cortex (PCC), a posterior 'hub' of the default mode network (DMN) (Carhart-Harris et al., 2012; Berkovich-Ohana et al., 2016; Smigielski et al., 2019). Such inactivation has been compared to the disengagement of a 'reducing valve', leading to less constraints on the potential repertoire of brain activity configurations (Carhart-Harris et al., 2012; Carhart-Harris et al., 2014). We measured the entropy of narrow band EEG power fluctuations as a quantitative metric of such dysregulation. However, we note that entropy should not be used as an absolute proxy for the level of consciousness, since a very high level of entropy implies that the constituents of the system (e.g. neurons) behave independently in the statistical sense, preventing information integration. Giulio Tononi postulated that measuring the balance between the enhanced repertoire of states and the level of information integration could result in a more adequate metric for consciousness than entropy alone (Oizumi et al., 2014). The proposed metric ( $\square$ ) is difficult to compute, but can be approximated empirically by measuring EEG after a transcranial magnetic stimulation (TMS) pulse, a technique that could be used in future studies to investigate the meditative state (Casali et al., 2013).
Both long-term meditation (Sahdra et al., 2011; Strauss et al., 2014; Haukaas et al., 2018; Pokorski and Suchorzynska, 2018) and the acute effects of serotonergic psychedelics (Griffiths et al., 2016; Carhart-Harris et al., 2017; Carhart-Harris et al., 2018; Dos Santos et al., 2018; Garcia-Romeu and Richards, 2018; Palhano-Fontes et al., 2018) have been reported to enhance mood and well-being. Increased brain entropy has been reported during the acute effects of psychedelics, and was shown to predict changes in personality traits (Lebedev et al., 2016). Recently, Carhart-Harris and Nutt suggested that $5-\mathrm{HT}_{2 \mathrm{~A}}$ signaling mediates 'active coping', a process characterized by enhanced plasticity (Carhart-Harris and Nutt, 2017). According to this proposal, $5-\mathrm{HT}_{2 \mathrm{~A}}$ agonism increases the excitability of the brain or, figuratively, its 'temperature', drawing an analogy with a metal heated and subsequently cooled in order to increase its malleability (a process known as 'annealing'). Thus, the potential long-term benefits of $5-\mathrm{HT}_{2 \mathrm{~A}}$ agonism could relate to the increased flexibility induced by a transient state of higher entropy, potentiating brain plasticity (Carhart-Harris et al., 2014; Carhart-Harris and Nutt, 2017). The process of Vipassana meditation, which resulted in most of the EEG entropy increases observed in our study, can be compared to the process proposed by Carhart-Harris and Nutt. FA alone can lead to a state of 'dullness'; therefore, the excitation brought upon by distraction and the meta-awareness of such distraction is needed in equal measure. In other words, a balance between excitation and FA is required. Vipassana meditation can be conceptualized as a process of continuously increasing brain excitability and then reducing it after the distractor is examined and excluded from consciousness by the meditator. Future studies of gene expression (epigenetics) and positron emission tomography (PET) in expert Vipassana meditators should be conducted to identify whether this 'annealing' process is only useful as an analogy, or is indeed mediated by increased expression of the genes that encode $5-\mathrm{HT}_{2 \mathrm{~A}}$ receptors, as well as by higher levels of $5-\mathrm{HT}_{2 \mathrm{~A}}$ receptor density. Advances in the development of $5-\mathrm{HT}_{2 \mathrm{~A}}$ agonist $\mathrm{PET}$ radioligands, such as Cimbi-36 (Ettrup et al., 2016), represent a promising avenue of research to evaluate this hypothesis.

We note that while increased entropy has been reported for the acute effects of different compounds (e.g. 5- $\mathrm{HT}_{2 \mathrm{~A}}$ agonists and NMDA antagonists) (Schartner et al., 2017a), our results provide evidence of 'endogenous' increases, i.e. increased EEG entropy that is not mediated by pharmacological agents but by the mental processes executed by long-term meditators. Furthermore, our analyses identified the spectral bands that become more disorganized, matching those predominantly affected by $5-\mathrm{HT}_{2 \mathrm{~A}}$ agonists (alpha rhythm) (Muthukumaraswamy et al., 2013; Carhart-Harris et al., 2016) and by meditation in expert practitioners (gamma rhythm) (Lutz et al., 2004). While most of the studies to date investigated the entropic brain hypothesis from the perspective of broadband oscillations, our work puts forward novel results that should lead to a re-examination 
of brain states of higher entropy in terms of the affected frequency bands.

The observed changes in coherence and metastability suggest that the repertoire of cells recruited for bandspecific oscillations was locally increased during meditation while, at a more global scale, increased gamma band binding reduced such repertoire. This conflict between local and global changes in the potential repertoire of brain states is consistent with findings obtained under the acute effects of LSD, suggesting increased global functional integration at the expense of locally segregated activity (Tagliazucchi et al., 2016). Further electrophysiological studies using $5-\mathrm{HT}_{2 \mathrm{~A}}$ agonists and NMDA antagonists could be conducted to allow a direct comparison of local entropy and global metastability/coherence with those quantified during the meditative state.

Our study represents a step towards establishing an intuitive relationship between the enhancement of phenomenological space and increases in the entropy of brain activity, as proposed by Carhart-Harris et al. (2014). However, our study also presents limitations intrinsic to the use of EEG to infer the location of changes in entropy, since EEG electrode signals reaching the scalp from different cortical sources are 'mixed' due to volume conduction effects. Furthermore, while a psychometric assessment of the participants was available, it was not suitable for the comparison of phenomenological features with those of the psychedelic state. For this purpose, other questionnaires -such as the Altered States of Consciousness (ASC) questionnaire (Dittrich, 1998)should be employed. Due to this limitation, we can only provide indirect evidence of increased entropy as a mechanism underlying the phenomenological similarity between the meditative and the psychedelic states.

In summary, Vipassana meditation, a practice that can be classified as midway between $O A$ and $F A$, resulted in increased entropy of the envelope of brain oscillations in the alpha and low/high gamma bands. This suggested that the increased repertoire of local neural configurations was mediated by higher levels of local segregation alongside increased global coherence in the low/high gamma bands. Our study provides an example of a non-pharmacologically-induced brain state of high entropy. Future research should address whether these 'endogenous' increases in EEG entropy are related to changes in the expression of $5-\mathrm{HT}_{2 \mathrm{~A}}$ receptors in expert meditators, and study the relationship between entropy, flexibility, and the long-term positive effects that certain meditative traditions can have on physical and mental health.

\section{ACKNOWLEDGMENTS}

We thank Braboszcz and colleagues for sharing the EEG data together alongside their publication. We acknowledge insightful discussions with the members of the Psychedelic mailing list (Imperial College London). RMV and CT were supported by a CONICET postdoctoral fellowship. FZ was supported by a CONICET doctoral fellowship.

\section{REFERENCES}

Abraham A, Pedregosa F, Eickenberg M, Gervais P, Mueller A, Kossaifi J, Gramfort A, Thirion B, Varoquaux G (2014) Machine learning for neuroimaging with scikit-learn. Front Neuroinform 8:14.

Al-Angari HM, Sahakian AV (2007) Use of sample entropy approach to study heart rate variability in obstructive sleep apnea syndrome. IEEE Trans Biomed Eng 54(10):1900-1904.

Barrett FS, Griffiths RR (2018) Classic hallucinogens and mystical experiences: phenomenology and neural correlates. Curr Top Behav Neurosci 36:393-430.

Ben-Naim A (2012) Entropy and the second law: interpretation and missinterpretations. New Jersey: World Scientific.

Berkovich-Ohana A, Harel M, Hahamy A, Arieli A, Malach R (2016) Data for default network reduced functional connectivity in meditators, negatively correlated with meditation expertise. Data Brief 8:910-914.

Braboszcz C, Cahn BR, Levy J, Fernandez M, Delorme A (2017) Increased gamma brainwave amplitude compared to control in three different meditation traditions. PLoS One 12(1) e0170647.

Brandmeyer T, Delorme A, Wahbeh H (2019) The neuroscience of meditation: classification, phenomenology, correlates, and mechanisms. Prog Brain Res 244:1-29.

Breiman L (2001) Random forests. Machine Learn 45(1):5-32.

Bruce EN, Bruce MC, Vennelaganti S (2009) Sample entropy tracks changes in EEG power spectrum with sleep state and aging. J Clin Neurophysiol 26(4):257.

Buzsaki G (2006) Rhythms of the brain. Oxford University Press.

Cahn BR, Delorme A, Polich J (2010) Occipital gamma activation during Vipassana meditation. Cogn Process 11(1):39-56.

Carhart-Harris RL (2018) The entropic brain - revisited. Neuropharmacology 142:167-178.

Carhart-Harris RL, Erritzoe D, Williams T, Stone JM, Reed LJ, Colasanti A, Tyacke RJ, Leech R, Malizia AL, Murphy K, Hobden P, Evans J, Feilding A, Wise RG, Nutt DJ (2012) Neural correlates of the psychedelic state as determined by $\mathrm{fMRI}$ studies with psilocybin. Proc Natl Acad Sci USA 109(6):2138-2143.

Carhart-Harris RL, Nutt DJ (2017) Serotonin and brain function: a tale of two receptors. J Psychopharmacol 31(9):1091-1120.

Carhart-Harris RL, Leech R, Hellyer PJ, Shanahan M, Feilding A, Tagliazucchi E, Chialvo DR, Nutt D (2014) The entropic brain: a theory of conscious states informed by neuroimaging research with psychedelic drugs. Front Hum Neurosci 8:20.

Carhart-Harris RL, Roseman L, Bolstridge M, Demetriou L, Pannekoek JN, Wall MB, Tanner M, Kaelen M, McGonigle J, Murphy K, Leech R, Curran HV, Nutt DJ (2017) Psilocybin for treatment-resistant depression: fMRI-measured brain mechanisms. Sci Rep 7(1):13187.

Carhart-Harris RL, Bolstridge M, Day CMJ, Rucker J, Watts R, Erritzoe DE, Kaelen M, Giribaldi B, Bloomfield M, Pilling S, Rickard JA, Forbes B, Feilding A, Taylor D, Curran HV, Nutt DJ (2018) Psilocybin with psychological support for treatmentresistant depression: six-month follow-up. Psychopharmacology (Berl) 235(2):399-408.

Casali AG, Gosseries O, Rosanova M, Boly M, Sarasso S, Casali KR, Casarotto S, Bruno MA, Laureys S, Tononi G, Massimini M (2013) A theoretically based index of consciousness independent of sensory processing and behavior. Sci Transl Med 5 (198):198ra105.

Cavanna F, Vilas MG, Palmucci M, Tagliazucchi E (2017) Dynamic functional connectivity and brain metastability during altered states of consciousness. Neuroimage.

Chang D, Song D, Zhang J, Shang Y, Ge Q, Wang Z (2018) Caffeine caused a widespread increase of resting brain entropy. Sci Rep 8 (1):2700.

Dittrich A (1998) The standardized psychometric assessment of altered states of consciousness (ASCs) in humans. Pharmacopsychiatry 31(S 2):80-84.

Dos Santos RG, Bouso JC, Alcázar-Córcoles M, Hallak JEC (2018) Efficacy, tolerability, and safety of serotonergic psychedelics for 
the management of mood, anxiety, and substance-use disorders: a systematic review of systematic reviews. Expert Rev Clin Pharmacol 11(9):889-902.

Ettrup A, Svarer C, McMahon B, da Cunha-Bang S, Lehel S, Møller $K$, et al. (2016) Serotonin $2 A$ receptor agonist binding in the human brain with [11C] Cimbi-36: test-retest reproducibility and head-to-head comparison with the antagonist [18F] altanserin. Neuroimage 130:167-174.

Fries P (2009) Neuronal gamma-band synchronization as a fundamental process in cortical computation. Annu Rev Neurosci 32:209-224.

Fries P, Nikolić D, Singer W (2007) The gamma cycle. Trends Neurosci 30(7):309-316.

Garcia-Romeu A, Richards WA (2018) Current perspectives on psychedelic therapy: use of serotonergic hallucinogens in clinical interventions. Int Rev Psychiatry:1-26.

Griffiths RR, Johnson MW, Carducci MA, Umbricht A, Richards WA, Richards BD, Cosimano MP, Klinedinst MA (2016) Psilocybin produces substantial and sustained decreases in depression and anxiety in patients with life-threatening cancer: a randomized double-blind trial. J Psychopharmacol 30(12):1181-1197.

Haukaas RB, Gjerde IB, Varting G, Hallan HE, Solem S (2018) A randomized controlled trial comparing the attention training technique and mindful self-compassion for students with symptoms of depression and anxiety. Front Psychol 9:827.

Hudetz AG, Liu X, Pillay S, Boly M, Tononi G (2016) Propofol anesthesia reduces Lempel-Ziv complexity of spontaneous brain activity in rats. Neurosci Lett 628:132-135.

Kaffashi F, Foglyano R, Wilson CG, Loparo KA (2008) The effect of time delay on approximate \& sample entropy calculations. Physica D 237(23):3069-3074.

Kakumanu RJ, Nair AK, Venugopal R, Sasidharan A, Ghosh PK, John JP, Mehrotra S, Panth R, Kutty BM (2018) Dissociating meditation proficiency and experience dependent EEG changes during traditional Vipassana meditation practice. Biol Psychol 135:65-75

Koch C, Massimini M, Boly M, Tononi G (2016) Neural correlates of consciousness: progress and problems. Nat Rev Neurosci 17 (5):307.

Lakens D (2013) Calculating and reporting effect sizes to facilitate cumulative science: a practical primer for t-tests and ANOVAs. Front Psychol 4:863.

Lebedev AV, Kaelen M, Lövdén M, Nilsson J, Feilding A, Nutt DJ, Carhart-Harris RL (2016) LSD-induced entropic brain activity predicts subsequent personality change. Hum Brain Mapp 37 (9):3203-3213.

Lippelt DP, Hommel B, Colzato LS (2014) Focused attention, open monitoring and loving kindness meditation: effects on attention, conflict monitoring, and creativity - A review. Front Psychol 5:1083.

Liu X, Lauer KK, Ward BD, Roberts CJ, Liu S, Gollapudy S, Rohloff R, Gross W, Xu Z, Chen S, Wang L, Yang Z, Li SJ, Binder JR, Hudetz AG (2018) Regional entropy of functional imaging signals varies differently in sensory and cognitive systems during propofol-modulated loss and return of behavioral responsiveness. Brain Imaging Behav.

Luft CDB, Zioga I, Banissy MJ, Bhattacharya J (2019) Spontaneous visual imagery during meditation for creating visual art: an EEG and brain stimulation case study. Front Psychol 10

Lutz A, Dunne JD, Davidson RJ (2007) Meditation and the neuroscience of consciousness. In: Cambridge Handbook of Consciousness. p. 499-555.

Lutz A, Greischar LL, Rawlings NB, Ricard M, Davidson RJ (2004) Long-term meditators self-induce high-amplitude gamma synchrony during mental practice. Proc Natl Acad Sci USA 101 (46):16369-16373.

Lutz A, Slagter HA, Dunne JD, Davidson RJ (2008) Attention regulation and monitoring in meditation. Trends Cogn Sci 12 (4):163-169.

Muthukumaraswamy SD, Carhart-Harris RL, Moran RJ, Brookes MJ, Williams TM, Errtizoe D, et al. (2013) Broadband cortical desynchronization underlies the human psychedelic state. J Neurosci 33(38):15171-15183

Nikolić D, Fries P, Singer W (2013) Gamma oscillations: precise temporal coordination without a metronome. Trends Cogn Sci 17 (2):54-55.

Oizumi M, Albantakis L, Tononi G (2014) From the phenomenology to the mechanisms of consciousness: Integrated Information Theory 3.0. PLoS Comput Biol 10(5) e1003588.

Palhano-Fontes F, Barreto D, Onias H, Andrade KC, Novaes MM, Pessoa JA, Mota-Rolim SA, Osório FL, Sanches R, Dos Santos RG, Tófoli LF, de Oliveira Silveira G, Yonamine M, Riba J, Santos FR, Silva-Junior AA, Alchieri JC, Galvão-Coelho NL, LobãoSoares B, Hallak JEC, Arcoverde E, Maia-de-Oliveira JP, Araújo DB (2018) Rapid antidepressant effects of the psychedelic ayahuasca in treatment-resistant depression: a randomized placebo-controlled trial. Psychol Med Araújo:1-9.

Pincus SM, Goldberger AL (1994) Physiological time-series analysis: what does regularity quantify? Am J Physiol Heart Circ Physiol 266(4): $\mathrm{H} 1643-\mathrm{H} 1656$

Pokorski M, Suchorzynska A (2018) Psychobehavioral effects of meditation. Adv Exp Med Biol 1023:85-91.

Richman JS, Moorman JR (2000) Physiological time-series analysis using approximate entropy and sample entropy. Am J Physiol Heart Circ Physiol 278(6):H2039-2049.

Rosenthal R, Cooper H, Hedges L (1994) Parametric measures of effect size. Handbook Res Synth 621:231-244.

Sahdra BK, MacLean KA, Ferrer E, Shaver PR, Rosenberg EL, Jacobs TL, Zanesco AP, King BG, Aichele SR, Bridwell DA, Mangun GR, Lavy S, Wallace BA, Saron CD (2011) Enhanced response inhibition during intensive meditation training predicts improvements in self-reported adaptive socioemotional functioning. Emotion 11(2):299-312.

Schartner MM, Pigorini A, Gibbs SA, Arnulfo G, Sarasso S, Barnett L, Nobili L, Massimini M, Seth AK, Barrett AB (2017b) Global and local complexity of intracranial EEG decreases during NREM sleep. Neurosci Conscious 2017(1):niw022.

Schartner MM, Carhart-Harris RL, Barrett AB, Seth AK, Muthukumaraswamy SD (2017a) Increased spontaneous MEG signal diversity for psychoactive doses of ketamine, LSD and psilocybin. Sci Rep 7:46421.

Schartner M, Seth A, Noirhomme Q, Boly M, Bruno MA, Laureys S, Barrett A (2015) Complexity of multi-dimensional spontaneous EEG decreases during propofol induced general anaesthesia. PLoS One 10(8) e0133532.

Schoenberg P, Vago DR (2019) Mapping meditative states and stages with electrophysiology: concepts, classifications, and methods. Curr Opin Psychol 28:211-217.

Shanahan M (2010) Metastable chimera states in communitystructured oscillator networks. Chaos 20(1) 013108.

Smigielski L, Scheidegger M, Kometer M, Vollenweider FX (2019) Psilocybin-assisted mindfulness training modulates selfconsciousness and brain default mode network connectivity with lasting effects. Neuroimage 196:207-215.

Song Y, Crowcroft J, Zhang J (2012) Automatic epileptic seizure detection in EEGs based on optimized sample entropy and extreme learning machine. J Neurosci Methods 210(2):132-146.

Strauss C, Cavanagh K, Oliver A, Pettman D (2014) Mindfulnessbased interventions for people diagnosed with a current episode of an anxiety or depressive disorder: a meta-analysis of randomised controlled trials. PLoS One 9(4) e96110.

Tagliazucchi E, Carhart-Harris R, Leech R, Nutt D, Chialvo DR (2014) Enhanced repertoire of brain dynamical states during the psychedelic experience. Hum Brain Mapp 35(11):5442-5456.

Tagliazucchi E, Roseman L, Kaelen M, Orban C Muthukumaraswamy SD, Murphy $\mathrm{K}$, Laufs $\mathrm{H}$, Leech $\mathrm{R}$, McGonigle J, Crossley N, Bullmore E, Williams T, Bolstridge M, Feilding A, Nutt DJ, Carhart-Harris R (2016) Increased global functional connectivity correlates with LSD-induced ego dissolution. Curr Biol 26(8):1043-1050. 
Takens F (1981) Detecting strange attractors in turbulence. In Dynamical systems and turbulence, Warwick 1980 (pp. 366381). Springer, Berlin, Heidelberg.

Thomas JW, Cohen M (2014) A methodological review of meditation research. Front Psychiatry 5:74.

van Lutterveld R, van Dellen E, Pal P, Yang H, Stam CJ, Brewer J (2017) Meditation is associated with increased brain network integration. Neuroimage 158:18-25.

Venugopala RJ, Sasidharana A, Naira AK, Kutty BM, Ghosh PK (2017) Permutation Entropy as a Complexity Measure of EEG Time Series in Vipassana meditators. 23rd EEGLAB Workshop Jan 16-20 AlISH. Mysuru.

Viol A, Palhano-Fontes F, Onias $\mathrm{H}$, de Araujo DB, Viswanathan GM (2017) Shannon entropy of brain functional complex networks under the influence of the psychedelic Ayahuasca. Sci Rep 7 (1):7388.

Viol A, Palhano-Fontes $\mathrm{F}$, Onias $\mathrm{H}$, de Araujo DB, Hövel $\mathrm{P}$, Viswanathan GM (2019) Characterizing complex networks using entropy-degree diagrams: unveiling changes in functional brain connectivity induced by Ayahuasca. Entropy 21(2):128.

Yentes JM, Hunt N, Schmid KK, Kaipust JP, McGrath D, Stergiou N (2013) The appropriate use of approximate entropy and sample entropy with short data sets. Ann Biomed Eng 41(2):349-365.

Zhang X, Zhou P (2012) Sample entropy analysis of surface EMG for improved muscle activity onset detection against spurious background spikes. J Electromyo Kinesiol 22(6):901-907.

\section{APPENDIX}

\section{Computation of the SE}

The SE was developed to quantify the regularity or reproducibility in a time series by estimating the likelihood of observing patterns of length $m+1$ in the data within tolerance $r$ of each other, given that patterns of length $m$ are within tolerance $r$ of each other. In this definition, the parameter $\mathrm{m}$ is called the embedding dimension, a name inherited from the process of reconstructing an attractor from a time series by means of a discrete sequence of observations (Takens, 1981).

The procedure followed to compute SE is illustrated in Fig. 1B. We consider a time series of length $N$, given by $\left\{x_{1}, x_{2}, x_{3}, \cdots, x_{N}\right\}$, with homogeneous spacing between samples $(\tau)$, i.e. $\left|x_{i}-x_{j}\right|=\tau$. For the purposes of this analysis, the time series represents the instantaneous amplitude of the narrow band frequency envelopes obtained from the Hilbert transform. The template vectors of length $\mathrm{m}$ are defined as $X_{m}(i)=\left\{x_{i}, x_{i+1}, x_{i+2}, \cdots, x_{i+m-1}\right\}$. Given a suitable distance function $d$ (in this implementation, the Chebyshev distance), the distance between two template vectors of equal length is defined as $d\left(X_{m}(i), X_{m}(j)\right)$. Then, for a given embedding dimension $\mathrm{m}$ and tolerance $r$, we define the set $S_{m}(r)=\left\{i, j: d\left(X_{m}(i), X_{m}(j)\right) \leq r\right.$ and $\left.i \neq j\right\}$, i.e. all the pairs of different indices such that the associated template vectors are considered similar within a certain tolerance. Finally, we compute the SE as,

$\mathrm{SE}=-\log \left(\frac{\# S_{m+1}(r)}{\# S_{m}(r)}\right)$
(\# denotes set cardinality). We observe that, by construction, $S_{m+1}(r) \leq S_{m}(r)$, therefore $S E$ is equal or larger than zero. Also, we note that self-matches are not included in the computation of SE. Due to the exclusion of self-matches, SE results in a less biased estimate of entropy relative to other metrics (e.g. approximate entropy). Also, instead of matching the patterns against a fixed template, all possible segments of length $m$ are considered as templates, which increases the statistical power in Eq. (1). SE can be conceptualized as measuring the amount of self-similarity in the data: a highly regular and periodical time series will present a high SE, while a noisier and less predictable time series will present a lower SE.

In spite of its name, the embedding dimension is not estimated to guarantee the reconstruction of an attractor underlying the time series, but from practical considerations concerning the computation of the logarithmic likelihood in Eq. (1). As discussed by both Kaffashi et al. (2008), and Pincus and Goldberger (1994), the preferred choice $m=2$ allows a better estimate of the logarithmic likelihood, since $\# S_{m}(r)$ is a decreasing function of $m$. Concerning the tolerance, previous numerical studies showed that $r \leq 0.1 \times \mathrm{SD}$ leads to poor logarithmic likelihood estimates, while for $r \geq 0.25 \times \mathrm{SD}$ relevant information is lost. Therefore, we followed the recommendation of adopting an intermediate value $(r=0.2 \times \mathrm{SD})$.

\section{Metastability and coherence}

Consider $N$ coupled oscillators, each with its own characteristic oscillation frequency $\omega_{i}$. These frequencies represent those of the narrow-band filtered 64 EEG channel signals. The instantaneous amount of synchrony between the ensemble of oscillators can be measured with the Kuramoto order parameter,

$R(t)=\frac{1}{N}\left|\sum_{j=1}^{N} e^{i \theta_{j}}\right|$

The order parameter $R(t)$ ranges between 0 and 1 , indicating minimum and maximum synchrony, respectively. From Eq. (2) we derive the metastability $\mathrm{K}$ as the variance of $R(t)$, indicating the amplitude of the dynamical repertoire of the oscillators (i.e. the occurrence of transient synchronization and desynchronization over time), and the coherence $\mathrm{C}$ as the mean of $R(t)$, indicating the average level of synchronization between the oscillators.

As outlined in Fig. 1C of the main manuscript, Eq. (2) can be used to investigate the collective behavior of the 64 EEG channels recorded from the different groups of subjects. In this case, the characteristic oscillation frequencies $\omega_{i}$ are determined by the bandpass filtering, and the instantaneous phases $\theta_{j}$ are obtained from the Hilbert transform. 